\title{
Imnuno-Oncología: Recuento Histórico y Fundamentos Básicos.
}

\section{Imnuno-Oncology: Historical Recount and Basic Fundamentals.}

\section{Verónica Guerra Cevallos', Solange Núñez-González², Estefanía Ochoa², Camilo Félix1, Daniel Simancas-Racines* iD.}

1. Universidad Tecnológica Equinoccial, Facultad de Ciencias de la Salud Eugenio Espejo. Quito-Ecuador.

*Correspondencia:

dsimancas@ute.edu.ec

Teléfono [593] 022990800

Conflicto de intereses: Los autores declaran no tener conflictos de intereses.

Fondos: Ver la página 70

Recibido: 5 Julio 2017

Aceptado: 20 Diciembre 2017

Publicado: 30 Abril 2018

Membrete bibliográfico:

Guerra V, Núñez-González S,

Ochoa E, Félix C, Simancas-

Racines D. Inmuno-Oncología:

Recuento Histórico y

Fundamentos Báscios. Rev. Oncol.

Ecu 2018;28(1):62-72.

DOI: https://doi.org/10.33821/213

Copyright Guerra, et al. Este artículo es distribuido bajo los términos de Creative Commons Attribution License, el cual permite el uso y redistribución citando la fuente y al autor original.
2. Centro de Investigación en Salud Pública y Epidemiología Clínica (CISPEC). Facultad de Ciencias de la Salud Eugenio Espejo. Universidad Tecnológica Equinoccial. Quito-Ecuador .

\section{Resumen}

El sistema inmune cumple un rol fundamental en la defensa contra microorganismos y células anómalas. Históricamente, el concepto de vigilancia inmunológica se fundamenta en el control de múltiples funciones incluyendo la regulación de células cancerígenas a través de diversos mecanismos, en los cuales están involucrados: células, moléculas y tejidos del sistema inmune. El objetivo de analizar la respuesta inmune frente al cáncer, es entender los mecanismos de presentación del antígeno y los mecanismos desencadenados por el sistema adaptativo e innato que participan en la destrucción del tumor a expensas de un proceso inflamatorio agudo que podría llevar al control o destrucción del cáncer. La propuesta de esta revisión es resumir y esquematizar los aspectos cardinales de los diferentes procesos inmunológicos que participan en la fisiopatología de las enfermedades malignas, así como los mecanismos que emplea el sistema inmune para la defensa del cáncer.

Palabras Claves: SISTEMA INMUNOLÓGICO, INMUNIDAD, FORMACIÓN DE ANTICUERPOS, ANTÍGENOS, ANTÍGENOS CD8, ANTÍGENOS CD4, MONITORIZACIÓN INMUNOLÓGICA.

DOI: $10.33821 / 213$

\section{Abstract}

The Inmune System plays an essential role in the defense of the organism against microorganisms and alters cells. Historically, the concept of immune surveillance its based in the control of multiple functions including the regulation of cancer cells through diverse mechanisms such as cells, molecules and tissue from the immune system. Therefore, it is important to understand the mechanisms of antigen presentation and other mechanisms of the innate and adaptive system which participate in the defense of the organism against the tumor. This process is enhancing by an 
inflammatory acute process that could lead to the control or de destruction of the tumor. The purpose of this review is to develop the cardinal aspects of the immunologic process that take part in the defense against malignant diseases, and also to explain its mechanisms.

Keywords: IMMUNE SYSTEM, IMMUNITY, FORMATION OF ANTIBODIES, ANTIGENS, CD8 ANTIGENS, CD4 ANTIGENS, IMMUNE MONITORING.

DOI: $10.33821 / 213$

\section{Introducción}

Al hablar de Inmunología como la ciencia que estudia los mecanismos de defensa, y asociarla con el cáncer, en un contexto vinculado a "malignidad", es imperativo conocer y entender los mecanismos que emplea el sistema inmune y como éste actúa frente al cáncer.

Normalmente, existe un equilibrio entre el proceso de renovación y el de muerte celular (apoptosis), estos dos procesos son de gran trascendencia en nuestro cuerpo para el mantenimiento de las células, es decir, las células deterioradas de nuestro cuerpo deben morir y renovarse por nuevas células. Se considera que en el trascurso de la vida esta renovación es de $10^{16}$ divisiones celulares [1].

En el proceso de división celular, la replicación del Ácido Desoxirribonucleico (ADN) origina dos copias idénticas del genoma diploide, durante la división puede ocurrir de forma esporádica mutaciones, generando cambios en el $A D N$, con el consecuente daño químico en el mismo. Pese a todos los mecanismos de control enzimático, si esta mutación ocurre en células somáticas se manifestará en el individuo que fue afectado [1,2], si la mutación genera una variación entre el proceso de división y supervivencia de las células, se producirán alteraciones funcionales del órgano, dando origen a tumores. Si el tumor no invade a los tejidos vecinos y está encapsulado se lo denomina "benigno", pero si este invade los tejidos se denomina maligno o cáncer [2].

Los relatos históricos de los tumores y/o cánceres se vienen mencionando desde tiempos antes de Cristo; especialmente sobre su origen y posibles tratamientos. En los papiros de Smith describe la cirugía del cáncer, mientras que en el de Ebers detalla el tratamiento farmacológico y mecánico del cáncer [3]. En el siglo IV a.C. con Hipócrates, surge el origen etimológico de la palabra cáncer, descritas como lesiones ulcerosas, algunas veces endurecidas que se desarrollan progresivamente sin control, y que se expanden hacia los tejidos semejantes a las patas de un cangrejo, por lo que lo denominó kapkívos, término griego que traducido al latín significa "cáncer"[4].

Hacia los años 25 a.C. - 50 d.C., aparece la palabra "tumor" del griego oykoc (oncos), utilizada para describir la hinchazón o edema, la misma se tradujo posteriormente al latín como tumor y fue Celso quien lo asocia con uno de los signos clásicos de inflamación [5].

De 129-157 d.C., Galeno describe a los tumores dentro de un concepto humoral, siendo estas lesiones producto de alteraciones del humor "bilis negra", y la misma debía ser eliminada por cirugía o cauterización con fuego [6]. Varios años duró la caracterización de 
estas lesiones hasta que en el siglo XIX, con el aparecimiento del microscopio, se descubre que las células cancerosas tienen diferente morfología en relación a las normales.

En 1865 Virchow, demostró que una célula proviene de otra y que las células cancerosas debían derivar de otras con un estado similar, además la relación entre cáncer e inflamación, considerando a la inflamación como una irritación del estroma en el cual aparece la célula neoplásica [7]. En el siglo XX, las investigaciones se focalizaron en la célula cancerosa y en el cáncer como una enfermedad genética, Muller en 1927 tras realizar varios trabajos experimentales confirmó la capacidad de los rayos $\mathrm{X}$ para provocar mutaciones.

En 1761, Hill y Plott (1775), reportan la relación entre la inhalación del tabaco en polvo y el cáncer oral, así como el cáncer de escroto en deshollinadores de chimeneas [8]. Se ha considerado que la asociación del cáncer con diversos agentes químicos fue creciendo con el desarrollo industrial [9].

\section{Defendiéndonos contra el Cáncer}

El sistema inmune se encarga de brindarnos protección contra agentes patógenos y células propias modificadas o alteradas, para lo cual utiliza los mecanismos de inmunidad innata y adaptativa, jugando un papel importante en la defensa contra el cáncer [10].

Estos conceptos ya los mencionó Paul Ehrlich en 1900, quien postuló que existen moléculas encargadas de reconocer y destruir tumores [11]. Hacia 1970, Burnet, insta la Teoría de la Vigilancia Inmunológica, indicando que generalmente se producen células cancerosas en el cuerpo, que corresponde a las mutaciones celulares, pero que el sistema inmune es capaz de eliminarlas por reconocerlas como extrañas $[12,13]$.

En esencia propone que el sistema inmune reconoce y elimina clones de células neoplásicas que aparecen en el organismo, a partir del reconocimiento de antígenos del tumor [14]. A mediados del siglo XX, esto fue sustentado por Lewis Thomas, quien además menciona que la eliminación de las células cancerosas lo realiza el sistema inmune celular, sin embargo, esta teoría fue abandonada por datos poco sustentables hacia 1970 [15].

A partir de 1990 la función de la inmunovigilancia nuevamente vuelve a reactivarse, por las observaciones en individuos inmunodeprimidos (pacientes trasplantados o en uso de inmunosupresores) en los que se detectó una mayor probabilidad de presentar cáncer, es así que en los pacientes sometidos a trasplantes de pulmón y/o corazón, que recibieron tratamiento inmunosupresor, se detectó con mayor frecuencia, 7.1 más casos de neoplasias, en comparación con la población general [16].

A esta teoría se suma la evidencia observada en pacientes con inmunodeficiencias primarias y en pacientes infectados con el virus del $\mathrm{VIH}$, quienes son más propensas a desarrollar ciertos tipos de cáncer que las personas sin la infección o inmunodeficiencias [17], por tanto, si el sistema inmune no puede reconocer las células cancerosas, éstas van a persistir e invadir. Se ha demostrado que la presencia de linfocitos en el infiltrado de 
tumores primarios constituye un factor de pronóstico para la supervivencia de los pacientes [15].

En diciembre de 1971, el Instituto Nacional del Cáncer de los Estados Unidos (NCl), crea el Programa Nacional del Cáncer, enfocado en la investigación para el conocimiento de las bases científicas del cáncer, de tal forma que los objetivos propuestos por este grupo fueron: disminuir la incidencia, la morbilidad y la mortalidad del cáncer $[18,19]$.

Los adelantos realizados en el funcionamiento del sistema inmune, han permitido entender la presentación antigénica por células dendríticas, la interacción de las moléculas participantes en el reconocimiento y activación de los linfocitos, la actividad citotóxica de las células citolíticas y células asesinas naturales (por sus siglas en Inglés NK), la acción del Interferon gamma (IFN- $\gamma$ ) en la respuesta contra tumores, entre otros mecanismos involucrados en la defensa del cáncer [20].

Dentro de las investigaciones sobre la naturaleza del cáncer, se ha considerado como una enfermedad genética, puesto que cumple con las siguientes características [21]:

1) Capacidad de señalización para una proliferación mantenida.

2) Resistencia de las células para la muerte.

3) Capacidad de inducir angiogénesis.

4) Inmortalidad de las células.

5) Capacidad de invasión y metástasis.

6) Falla en la supresión del crecimiento.

\section{Respuesta inmune en el Cáncer}

La función del sistema inmune en la vigilancia de los tumores, fue considerada inicialmente de forma exclusiva para impedir el crecimiento de células malignas, los linfocitos $T$ citotóxicos (CTL) y NK son sustanciales para la eliminación del tumor [22, 23]. En la actualidad, se sabe que la relación entre las células del sistema inmune y las células tumorales es más compleja, con procesos dinámicos en los cuales las mismas células del ambiente tumoral de defensa son promotoras del tumor [24].

Al establecerse el tumor, estos adquieren capacidades para prevenir la respuesta antitumoral, es decir escapan de la respuesta inmune con la pérdida de los antígenos por disminución de moléculas encargadas de la presentación del antígeno (por sus siglas en Inglés MHC) o pérdida de la ruta de presentación del antígeno, supresión inmune a cargo de citocinas o interacciones directas entre ligandos tumorales y receptores de células inmunes, contribuyendo a la inmuno-edición, en el que las células tumorales resisten, proliferan y se desarrollan, por tanto se promueve el crecimiento del tumor y la metástasis [25].

Dos interrogantes importantes que requieren respuesta entre la relación sistema inmune y cáncer, son: ¿cómo el sistema inmune es capaz de destruir las células tumorales producto 
de una inflamación aguda?, y ¿cómo la respuesta inflamatoria crónica promueve el crecimiento del tumor más que su eliminación? [26]; el estudio de esta respuesta inmune al cáncer, sustenta la teoría de la inmunovigilancia [27].

En modelos animales, se establece que el reconocimiento inicial del sistema inmune al tumor se denomina inmuno-edición, mismo que puede desencadenar tres desenlaces [20]:

1.- Eliminación del cáncer.

2.- Equilibrio del cáncer debido a la selección inmune de los tumores menos inmunogénicos.

3.- Escape del tumor con un consecuente crecimiento, resistiendo a la destrucción por parte del sistema inmune.

Para que exista una respuesta inmune eficiente, el estímulo provocado por los antígenos debe ser importante, los antígenos tumorales, pueden ser el producto de varios mecanismos como: la sobreexpresión de proteínas en la superficie de las células, el resultado de mutaciones genéticas o modificaciones pos-traduccionales (postraslation), los productos de infecciones virales (virus oncógenicos), los antígenos oncofetales como el carcino embrionario, alfafetoproteína, alteraciones originadas por compuestos químicos, entre otros [28].

Los antígenos extraños (oncovirus) al ser potentes inmunógenos, pueden despertar en el sistema inmune señales de alerta capaces de activar células de la inmunidad adaptativa e innata para iniciar la respuesta, no así las proteínas propias.

Uno de los objetivos del $\mathrm{NCl}$ de los Estados Unidos fue invertir en investigaciones que expliquen los mecanismos inmunológicos responsables en la defensa contra el cáncer, es por esto, que la comprensión de los conceptos de inmunología básica es indispensable para el entendimiento de todos los adelantos existentes en esta área [18].

A continuación se describirá en términos simples tres aspectos cardinales:

- ¿Cómo se realiza el reconocimiento de los antígenos tumorales?

- ¿Cuáles son las moléculas participantes en la activación de los linfocitos?

- ¿ ¿Cuáles son los mecanismos efectores para la destrucción del tumor?

\section{Reconocimiento de los Antígenos}

La respuesta inmune normal empieza a desarrollarse a partir del reconocimiento de un antígeno como extraño por parte del organismo, y en el caso de un proceso cancerígeno el reconocimiento es del antígeno tumoral. Los antígenos pueden ser exclusivos de estas células tumorales como es el caso de las proteínas mutadas o los antígenos asociados al tumor [20]. La mayoría de los casos de cáncer están localizados en células epiteliales, estas células al ser nucleadas sus antígenos proteicos serán expresados a través de Moléculas de Histocompatibilidad tipo I (MHC I), a diferencia de los antígenos exógenos que estarán expresados por moléculas de Histocompatibilidad tipo II (MHC II) [19, 28]. 
Partiendo de este concepto inicial, la destrucción de las células tumorales es realizada por los linfocitos T citolíticos (TCD8) de la inmunidad adaptativa [15]. La forma de reconocimiento de estos antígenos es de tipo indirecto o cruzado, para este tipo de reconocimiento, los antígenos tumorales que están en los órganos específicos, deben ser llevados a los ganglios linfáticos a través de células presentadoras de antígeno (CPA), por su capacidad de movilizar y procesar antígenos proteicos [28].

Las células dendríticas al ser CPAs, tienen la capacidad de procesar antígenos y lo realizan a través de moléculas MHC II y MHC I [29]; de tal forma que en el órgano linfático periférico (ganglios) las moléculas MHC II presentan a los linfocitos T auxiliares (TCD4) y las moléculas MHC I a los TCD8. La activación al linfocito T CD4, origina la producción de citoquinas como la interleuquina-2 (IL-2) y el IFN- $\gamma$ responsables de la activación y proliferación de linfocitos TCD8. Por tanto, es indispensable la función de los linfocitos TCD4 para la activación efectiva de los linfocitos TCD8 [20,21].

En base a lo anteriormente mencionado, cuando el individuo se encuentra en un estado de inmunosupresión, como en pacientes con SIDA o pacientes que utilizan medicación inmunosupresora aumentan el riesgo de desarrollar cáncer lo cual es consecuente a la disfunción de los Linfocitos TCD4, esto a su vez contribuye a fortalecer la teoría de la vigilancia inmunológica [19,25,28,30]. El sistema inmune a través de sus componentes celulares vigilarían a estas células malignas las eliminaría si no estuvieran afectadas, en los dos ejemplos anteriores, la cantidad o la función de los linfocitos TCD4 (en el SIDA, drogas inmunosupresoras) están afectadas [17], consecuentemente su contribución a base de citocinas no existe para la activación de los linfocitos $T$ citolíticos responsables de la eliminación de las células alteradas o con cáncer [15].

\section{Moléculas participantes en la activación de los linfocitos}

El encuentro entre la molécula MHC I que presenta el antígeno y el receptor del Linfocito T (TCR) encargado del reconocimiento, constituye la primera señal de la activación del linfocito $T$ [31]. La segunda señal lo realizan las moléculas accesorias como las coestimuladoras, las cuales se denominan B7 en la célula presentadora y CD28 en la molécula del linfocito $T$, cuya función es la producción de citocinas que ocasionan proliferación celular (IL-2) [20]. Otras moléculas presentes en la activación son: las moléculas CD40 de la CPA y la molécula CD40L en el linfocito, estas se encargan de intensificar la acción de la CPA con aumento de la expresión de B7, de moléculas MHCII. Estas moléculas mejoran la presentación antigénica y la producción de citocina IL-12 encargada de proporcionar el comando para la producción de un patrón de citocinas Th1 colaborador con IFN-Y $[19,20]$.

Así también, existen otras moléculas que participan en la activación de los linfocitos, las encargadas de la traducción de señal, denominadas CD3 y $\zeta$, y moléculas que permiten la adherencia intercelular el ICAM-1 en la CPA y la integrina Antígeno de Función Linfocitaria1 (por sus siglas en Inglés LFA-1) en el linfocito, estas moléculas contribuyen para completar la señalización intracelular, como también la adhesión intercelular para fijar a estas células durante este proceso [28].

En la respuesta inmune al cáncer, también se incluye la activación de linfocitos B para la producción de anticuerpos. Este tipo de activación es similar a lo mencionado anteriormente, pues los antígenos al ser proteicos requieren colaboración entre linfocitos 
T y $\mathrm{B}$, con producción de inmunoglobulina IgG que participarán en la respuesta efectora. Las células del sistema inmune innato que se encargan de reconocer células anómalas en el cáncer son los NK, que son capaces de reconocer células que han perdido la capacidad de expresar moléculas de MHC-I, a través de receptores que presentan lípidos (CD1d) [31]; estas células participan de la vigilancia inmunológica.

La función que cumplen las células NK es trascendental, pues trabajan como células efectoras citolíticas por vía enzimática o a través de receptores Fas- FasL [21,32], además, producen citoquinas como el IFN- $\gamma, \mathrm{IL}-2, \mathrm{II}-4, \mathrm{IL}-10, \mathrm{IL}-13, \mathrm{IL}-17, \mathrm{II}-21, \mathrm{IL}-22$, factor estimulante de colonia de granulocitos y monocitos (GM-CSF), factor de necrosis tumoral alfa (TNF-a) siendo capaces de activar otras células como linfocitos T, B [33] y potenciar la cascada de citocinas y quimoquinas. Así, también contribuye a la maduración de las células dendríticas importantes para la presentación de antígenos [34].

\section{Respuesta efectora del sistema inmune}

Una vez que los linfocitos están activados, se produce la respuesta inmune efectora para la destrucción de las células cancerígenas, la acción citolítica de los Linfocitos TCD8 específicos y la acción de las inmunoglobulinas especialmente lgG en conjunto con otros mecanismos humorales como el complemento y mecanismos celulares con linfocitos NK producen citotoxicidad dependiente de anticuerpos. Los linfocitos NK son activados por citoquinas como IL-2, y son transformados en NK activadas por citoquinas (LAK), que aumentan su función citolítica [19, 21, 23].

La liberación de citocinas inflamatorias atrae otras células al tumor como macrófagos (M1) activados clásicamente por IFN- $\gamma$, con funciones microbicidas especialmente la liberación de enzimas lisosómicas, productos intermediarios de oxígeno, y nitrógeno, y la producción de citocinas como TNF-alfa. El TNF, localmente atrae células inflamatorias y desencadena una actividad en cascada con liberación de IL-1, IL-6 y quimoquinas. Consecuentemente, esto ocasiona un fenómeno inflamatorio agudo localizado que contribuirá con la eliminación delas células [19].

\section{Inmunoedición: Eliminación, equilibrio y Escape.}

La respuesta inmune efectora en pacientes inmunocompetentes, cumplen tres funciones: limitación del crecimiento del tumor, escape y los mecanismos de escape de la respuesta ${ }^{21}$. La primera función es la de focalizar y eliminar el tumor o la contracción del mismo. Así, cuando existe un crecimiento lento la respuesta se acompaña de una reactivación de la respuesta inmune destruyendo nuevamente el crecimiento cancerígeno, o a su vez originan una supresión de marcadores en la superficie de la célula tumoral originando una fase de equilibrio o latencia. En la fase de equilibrio, el tumor está presente pero no hay enfermedad clínica, siendo este periodo largo y simulando eliminación [19]. 
Otra posibilidad es el escape de los mecanismos inmunológicos, en las que se producen citocinas inmunosupresoras y existe incapacidad para presentar antígenos por falta de disponibilidad de moléculas de histocompatibilidad. Consecuentemente, no se desarrolla un reconocimiento o una respuesta efectora capaz de eliminar al tumor. Las mismas células efectoras contribuyen a esta fase produciendo citocinas inmunosupresoras que van a modular la respuesta en contra del huésped. La fase de equilibrio y de los mecanismos de escape de la respuesta se discutirán posteriormente [19, 21].

\section{Conclusiones}

El estudio del cáncer con una visión inmunológica ha permitido desarrollar diferentes conceptos como el de la respuesta inmune normal hasta el desarrollo celular de carácter maligno celular. El descubrimiento de la formación de antígenos tumorales, el reconocimiento de los antígenos que llevarán a montar una respuesta eficiente con células citolíticas, la identificación de componentes de comunicación celular como las citoquinas son conceptos que fueron desarrollados a través del estudio del Sistema Inmune y han sido de gran beneficio para el entendimiento de cuadros clínicos con carácter de malignidad. A pesar de los grandes avances en este campo, es fundamental desarrollar más estudios para dilucidar conceptos que podrían aportar en el manejo y tratamiento de estas patologías.

\section{Agradecimientos}

Reconocemos a las personas que ayudaron a la compilación bibliográfica de los artículos en la presente revisión, en especial al personal de la biblioteca de Facultad de Ciencias de la Salud Eugenio Espejo de la Universidad Tecnológica Equinoccial.

Nota del Editor

La Revista Oncología Ecu permanece neutral con respecto a los reclamos jurisdiccionales en mapas publicados y afiliaciones institucionales.

\section{Información adicional}

\section{Abreviaturas}

ADN: Ácido Desoxirribonucleico.

CPA: Células presentadoras de antígenos.

GM-CSF: Factor estimulante de colonia de granulocitos y monocitos.

IFN-Y: Interferon gamma.

LTC: Linfocitos T citotóxicos.

MCH: Moléculas de Histocompatibilidad.

MHC: Moléculas presentadoras de antígenos.

NCl: Instituto Nacional del Cáncer (Estados Unidos).

NK: Natural Killer.

SIDA: Síndrome de inmunodeficiencia adquirida.

TCD8: linfocitos T citolíticos.

TCR: Receptor del linfocito T. 
TNF-alfa: Factor de necrosis tubular alfa.

\section{Archivos Adicionales}

Ninguno declarado por los autores.

\section{Fondos}

Los fondos de la investigación fueron propios de los autores del presente artículo.

\section{Disponibilidad de datos y materiales}

Existe la disponibilidad de los artículos consultados en la presente revisión.

\section{Contribuciones de los autores}

Todos los autores participaron por igual en la concepción de la idea de revisión, recolección de artículos, escritura del artículo. Todos los autores leyeron y aprobaron la versión final del artículo.

Aprobación de ética y consentimiento para participar

No aplica a un artículo de revisión.

\section{Consentimiento para publicación}

No aplica.

\section{Referencias}

Abreviaturas en la referencias

DOI: Digital Object

Identifier

PMID: PubMed Identifier

SU: Short URL
1. Muñoz A. Origen y causas del cáncer. En: Editorial Hélice. Cáncer: genes y nuevas terapias. Madrid; 1997; p.6-23. SU: bit.ly/2RS1noi

2. Parham P. Manipulación de la respuesta inmunitaria. En: Editorial Médica Panamericana. Inmunología. Buenos Aires; 2006; p.449-468

3. López J. Una rareza bibliográfica universal: el Papiro médico de Edwin Smith. ACIMED [revista en la Internet] 2002;10(3):9-10. SU: bit.ly/2SdHaZp 
4. Salaverry O. La etimología del cáncer y su curioso curso histórico. Rev Peru Med Exp Salud Pública. 2013; 30(1):137-41. SU: bit.ly/2UpRVFA

5. Keil $\mathrm{H}$. The historical relationship between the concept of tumor and the ending -oma. Bulletin of the History of Medicine. 1950;24(4):352- 377.

6. Barrera V, Manero R. Medicina, cáncer y significación del cuerpo. TRAMAS 32 UAM-X México. 2009; 121-146.

7. Balkwill F, Mantovani A. Inflammation and cancer: back to Virchow? Lancet. 2001; 357(9255):539-545. DOI: $10.1016 /$ S0140-6736(00)04046-0

8. Waldron H. A brief history of scrotal cancer. Br J Ind Med. 1983;40(4):390-401. SU: bit.ly/2WsGuPk

9. Danaei G, Vander Hoorn S, Lopez AD, Murray CJ, Ezzati M. Causes of cancer in the world: comparative risk assessment of nine behavioral and environmental risk factors. Lancet. 2005;366(9499):1784-93. DOI: $10.1016 /$ S0140-6736(05)67725-2

10. Schreiber R, Old L, Smyth M. Cancer immunoediting: integrating immunity's roles in cancer suppression and promotion. Science. 2011;331(6024):1565-70. DOI: 10.1126/science.1203486.

11. Chowa M, Möllerb A, Smyth M. Inflammation and immune surveillance in cancer. SeminCancer Biol. 2012;22(1):23-32. DOI: 10.1016/j.semcancer.2011.12.004

12. Burnet F. Immunological surveillance in neoplasia. Transplant Rev. 1971;7:3-25. SU: $\underline{\text { bit.ly/2RRt948 }}$

13. Burnet F. The concept of immunological surveillance. ProgExp Tumor Res. 1970;13:1-27. DOI: $\underline{10.1159 / 000386035}$

14. Rabinovich G, Gabrilovich D, Sotomayor E. Immunosuppressive Strategies that are Mediated by Tumor Cells. AnnuRevImmunol. 2007;25:267-96. DOI: 10.1146/annurev.immunol.25.022106.141609

15. Stephen F, Dranoff G. The biologic importance of Tumor-infiltrating Lynphocytes. J Cutan Pathol. 2010;37(01):48-53. DOI: 10.1111/j.1600-0560.2010.01506.x

16. Roithmainer S, Haydon AM, Loi S, Esmore D, Griffiths A, Bergin P, et al. Incidence of malignace in heart and/or lung transplant recipients: a single institution experience. J Heart Lung Transplant. 2007;26 (8):845-9. DOI:10.1016/j.healun.2007.05.019

17. Corthay A. Does the immune system naturally protect against cancer? .Frontirers in Immunology. 2014;5(197):1-8. DOI: 10.3389/fimmu.2014.00197

18. De Vitta V, Rosenberg S. Two hundred years of cancer research. N Engl J Med. 2012;366(23):2207-14. DOI: $10.1056 /$ NEJMra1204479.

19. Burkholder B, Huang R, Burgess R, Luo S, Jones V, Zhang W, et al. Tumor-induced perturbations of cytokines and immune cell networks. Biochimica Biophys Acta. 2014;1845(2):182-201. DOI: 10.1016/j.bbcan.2014.01.004.

20. Finn O. Cancer Immunology. N Engl J Med 2008;358:2704-2715. DOI: 10.1056/NEJMra072739

21. Finn 0. Immuno-oncology: understanding the function and dysfunction of the immune system in cancer. Annals of Oncology. 2012;23 (Suppl 8):viii6-viii 9. DOI: 10.1093/annonc/mds256.

22. Marcus $A$, Gowen $B$, Thompson $T$, lannello A, Ardolino $M$, Deng $W$, et al.Recognition of tumor by innate immune system and natural killer cells. Adv Immunol. 2014;122:91-128. DOI: 10.1016/B978-0-12800267-4.00003-1.

23. Ryan C, Schell T. Accumulation of CD8+ T Cells in Advanced-Stage Tumors and Delay of Disease Progression following Secondary Immunization against an Immunorecessive Epitope. J Immunol 2006;177(1):255-267. DOI: 10.4049/jimmunol.177.1.255 
24. Coussens L, Werb Z. Inflammation and cancer. Nature. 2002;420(6917):860-867. DOI: $10.1038 /$ nature01322

25. Reiman J, Kmieciak M, Manjili M, Knutson K. Tumor Immunoediting and Immunosculpting Pathways to Cancer Progression. Semin Cancer Biol. 2007;17(4):275-287. DOI: 10.1016/j.semcancer.2007.06.009

26. Smith A, Kang Y. The Metastasis-Promoting Roles of Tumor-Associated Immune Cells. J Mol Med (Berl). 2013;91(4):411-429. DOI: 10.1007/s00109-013-1021-5

27. Bruttel V, Wischhusen J. Cancer stem cell immunology: key to understanding tumorigenesis and tumor immune escape? Front Immunol. 2014;5:360. DOI: 10.3389/fimmu.2014.00360

28. Abbas A, Lichtman A, Pillai S. Inmunología celular y molecular. Séptima edición. Elsevier Saunders. España. 2012; 389-405.

29. Vacchelli E, Vitale I, Eggermont A, Fridman WH, Fučíková J, Cremer I, et al. Trial watch: Dendritic cellbased interventions for cancer therapy. Oncoimmunology. 2013;2(10):e25771. DOI: 10.4161/onci. 25771 .

30. Robertson C, Berzofsky A, Terabe M. NKT cell networks in the regulation of tumor immunity. Front Immunol. 2014;5(543):2-12. DOI: 10.3389/fimmu.2014.00543

31. Van Der Merwe V, Dushek O. Mechanisms for T cell receptor triggering. Nat Rev Immunol. 2011;11(1):4755. DOI: $10.1038 /$ nri2887

32. Cheng M, Chen $Y$, Xiao W, Sun R, Tian Z. NK cell-based immunotherapy for malignant diseases. Cellular\& Molecular Immunology. 2013;10(3):230-252. DOI: 10.1038/cmi.2013.10.

33. Lang Mark L. How do natural killer T cells help B cells? Expert Rev Vaccines. 2009;8(8):1109-1121. DOI: $\underline{10.1586 / \text { erv. } 09.56}$

34. Dougan M, Dranoff G. Inciting inflammation: the RAGE about tumor promotion. J Exp Med. 2008; 205(2):267-270. DOI: 10.1084/jem.20080136 